Methods: Serum samples from patients enrolled in the Pathobiology of Early Arthritis Cohort (PEAC) were collected before treatment with MTX. Response to therapy was determined after 6 months by calculating the initial and final DAS28 of the patients. Their classification was performed following the EULAR response criteria. Sixty samples at baseline from this cohort (30 good responders and 30 non-responders) were depleted from the 14 most abundant proteins by affinity chromatography to remove background. Then, they were analysed by reversedphase nanoliquid chromatography coupled to mass spectrometry using a SWATH strategy in a tripleTOF MS (Sciex). The quantitative data obtained in this proteomic analysis were processed using the ProteinPilot 5.0.1 and PeakView 2.1 software (Sciex). Machine learning analyses were performed on a train set of 30 samples (15 responders and 15 non-responders) via support vector machine (SVM) using the Classyfire, e1071 and caret R packages. Results were verified in an independent set of 24 samples by a two-stage support vector machine (TSSVM) with RBF kernel and 10 cross-fold validation for each meta-model.

Results: The proteomic analysis led to the identification and quantification of 229 proteins that were common between the screening and validation sets. Independent screening and validation data sets were preprocessed by PCA for dimension reduction and analysed by machine learning tools, leading to the definition of a panel of 8 proteins (one of them involved in MTX metabolism) differentiating the groups of responders and non-responders to MTX with strong agreement (Kappa $>0.80$ ), very high accuracy and good relevant metrics (table 1).

Abstract SAT0074 - Table 1. Metrics of the classification performance of the 8-protein panel identified in this work to predict response of the patient to MTX. Cut-off for significance was $p$-value $<0.05$

\begin{tabular}{|c|c|c|c|c|c|c|c|}
\hline \multicolumn{8}{|l|}{ Train set } \\
\hline Accuracy & $95 \% \mathrm{Cl}$ & p-value & Kappa & Sensitivity & Specificity & $\begin{array}{l}\text { Pos } \\
\text { pred } \\
\text { value }\end{array}$ & $\begin{array}{l}\text { Neg } \\
\text { pred } \\
\text { value }\end{array}$ \\
\hline 0.9333 & $\begin{array}{c}(0.7793 \\
- \\
0.9918)\end{array}$ & $1.108 \mathrm{e}-05$ & 0.8667 & 0.8824 & 1.0000 & 1.0000 & 0.8667 \\
\hline \multicolumn{8}{|c|}{ Validation set } \\
\hline Accuracy & $95 \% \mathrm{Cl}$ & p-value & Kappa & Sensitivity & Specificity & $\begin{array}{l}\text { Pos } \\
\text { pred } \\
\text { value }\end{array}$ & $\begin{array}{c}\text { Neg } \\
\text { pred } \\
\text { value }\end{array}$ \\
\hline 0.9583 & $\begin{array}{c}(0.7888 \\
- \\
0.9989)\end{array}$ & 0.0007722 & 0.9091 & 1.0000 & 0.9375 & 0.8889 & 1.0000 \\
\hline
\end{tabular}

Conclusions: We have defined a panel of circulating proteins useful to predict the response to MTX therapy in rheumatoid arthritis patients.

Disclosure of Interest: None declared

DOI: 10.1136/annrheumdis-2018-eular.3433

\section{SAT0075 ADDITIONAL TARGET OF NORMAL SERUM MATRIX METALLOPROTEINASE-3 IS A POTENTIAL BIOMARKER FOR LESS ONE-YEAR RADIOGRAPHIC PROGRESSION IN RHEUMATOID ARTHRITIS}

L.-F. Chen, J.-D. Ma, Y.-Q. Mo, X.-Y. Du, D.-H. Zheng, L. Dai. Department of Rheumatology, Sun Yat-Sen Memorial Hospital, Sun Yat-Sen University, Guangzhou, China

Background: Matrix metalloproteinase (MMP) -3 plays important roles in bone and cartilage destruction in rheumatoid arthritis (RA). Our previous study showed continuously elevated serum MMP-3 for 3-6 months predict one-year radiographic progression in RA (Arthritis Res Ther. 2015 17:289). However, whether serum MMP-3 normalisation is a biomarker for better outcome remains elusive.

Objectives: To explore the association of serum MMP-3 normalisation with clinical and radiographic outcome in RA.

Methods: RA patients with moderate to high disease activity (DAS28-CRP>3.2) were treated according to treat to target (T2T) strategy and followed up at regular intervals $(0,1 \mathrm{st}, 3 \mathrm{rd}, 6$ th and 12th months). Demographic and clinical data were collected according to the 2017 EULAR recommendation and serum MMP-3 was detected by ELISA at each visit. X-ray assessment of hand/wrist was repeated at baseline and month 12 and radiographic progression was defined as a change of the Sharp/van der Heijde modified sharp score $\geq 0.5$ units.

Results: ( ${ }^{1}$ Among 200 RA patients recruited, there were $163(81.5 \%)$ female, with median disease duration $24\left({ }^{11-84}\right.$ months, median DAS28-CRP 4.9 (4.25.7). There were $29 \%$ patients showed one-year radiographic progression. ${ }^{2}$ The median MMP-3 was 209.7 (108.6-430.0) ng/ml. RA patients without radiographic progression had significant lower level of serum MMP-3 than those with radiographic progression at baseline and each visit (figure $1 \mathrm{~A}$, all $\mathrm{p}<0.001$ ). ${ }^{3}$ There were $13.0 \%, 14.5 \%, 17.0 \%, 25.5 \%$ and $31.0 \%$ patients having normal MMP-3 at baseline and $1 \mathrm{st}, 3 \mathrm{rd}, 6$ th and 12th months, respectively. There were significantly lower percentage of RA patients with normal MMP-3 at baseline and each visit showed radiographic progression than those with elevated MMP-3 (figure 1B, all $\mathrm{p}<0.05) .{ }^{4}$ There were $8.5 \%, 13.0 \%, 20.0 \%$ and $25.5 \%$ patients who achieved ther apeutic target and showed normal MMP-3 at 1 st, 3rd, 6th and 12th months, respectively. Among patients achieved therapeutic target, there were significantly lower percentage of normal MMP-3 patients showed radiographic progression than those with elevated MMP-3 (figure 1C, all $\mathrm{p}<0.05$ ). ${ }^{5}$ There were $6.5 \%$ $11.0 \%, 14.5 \%, 20.5 \%$ and $25.5 \%$ patients who had normal CRP and norma MMP-3 at baseline and $1 \mathrm{st}, 3 \mathrm{rd}$, 6th and 12th months, respectively. Among patients with normal CRP, there were significantly lower percentage of normal MMP-3 patients at $1 \mathrm{st}, 3 \mathrm{rd}, 6$ th and 12 th months showed radiographic progression than those with elevated MMP-3 (figure 1D, all $p<0.05$ ).
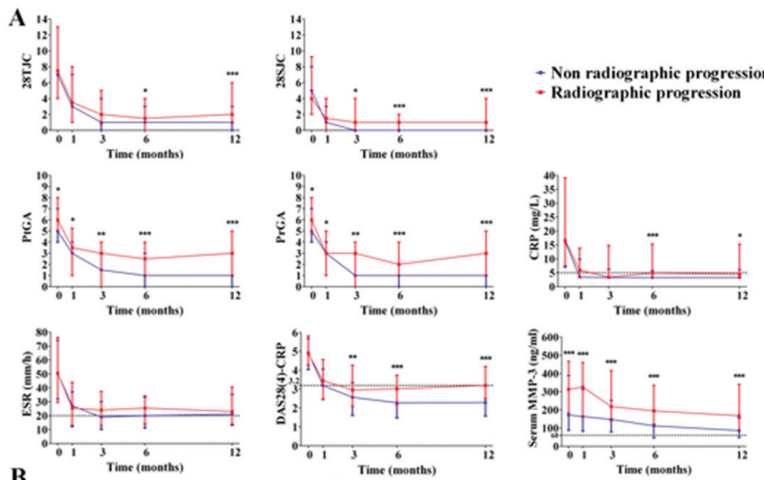

B
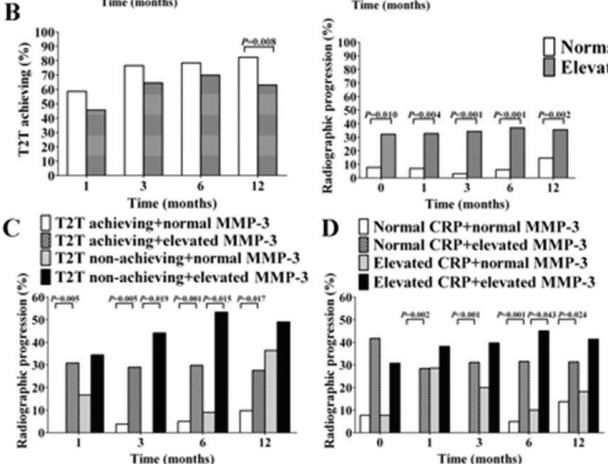

Abstract SAT0075 - Figure 1. Dynamic change of serum MMP-3 and indicators of disease activity and radiographic progression in RA. A Comparison of disease activity and radiographic progression indicators between RA patients with and without radiographic progression. B Comparison of T2T achieving and the percentage of RA patients showing radiographic progression between normal and elevated serum MMP-3 groups at each visit. C Comparison of the percentage of RA patients showing radiographic progression among groups with or without T2T achieving and normal serum MMP-3. D Comparison of the percentage of RA patients showing radiographic progression among groups with or without normal CRP and normal serum MMP-3.

Conclusions: Additional target of normal serum MMP-3 may be a potential biomarker for less one-year radiographic progression.

Acknowledgements: This work was supported by National Natural Science Foundation of China (no. 81471597 and 81671612), Guangdong Natural Science Foundation (no. 2016A030313307 and 2017A030313576) and Guangdong Medical Scientific Research Foundation (no. A2017093 and A2017109)

Disclosure of Interest: None declared

DOI: 10.1136/annrheumdis-2018-eular.4513

\section{SAT0076 \\ SYNOVIAL MAST CELLS AND RESPONSES TO SYNTHETIC AND BIOLOGIC DMARDS IN EARLY AND ESTABLISHED RHEUMATOID ARTHRITIS}

F. Rivellese, A. Nerviani, D. Mauro, S. Pagani, F. Humby, C. Pitzalis. Centre for Experimental Medicine and Rheumatology, William Harvey Research Institute, Barts and the London School of Medicine and Dentistry, London, UK

Background: Mast cells (MCs) are immune cells implicated in the pathogenesis of Rheumatoid Arthritis (RA), but their presence in synovia has not been assessed systematically and their association with disease progression and treatment response is unknown.

Objectives: To analyse MCs in the synovia of patients with early vs established RA in correlation with histological and clinical phenotype. 